\title{
Palatosquise em neonato de Fila Brasileiro
}

\author{
Cícero Rodrigues Feitosa Nunes ${ }^{1}$, Guilherme José Bolzani de Campos Ferreira ${ }^{2 *}$ \\ ${ }^{1}$ Médico Veterinário, Mestrando em Zootecnia da Universidade Federal do Piauí \\ ${ }^{2}$ Médico Veterinário, Docente do curso de Medicina Veterinária, Universidade Federal do Piauí \\ *Autor para correspondência, E-mail: guilherme.ferreira@ufpi.edu.br
}

\begin{abstract}
RESUMO. O presente relato visa descrever a alteração congênita de fenda palatina em um filhote de cão da raça fila Brasileiro com três dias de vida. A fenda palatina congênita é uma anomaliacongênita pouca frequente nos animais domésticos, caracterizada por um defeito de fusão tanto do palato primário, secundário ou ambos. A etiopatogenia desta anomalia ainda não é bem elucidada, entretanto, têm-se o conhecimento de várias possíveis causas, estas relacionadas aos fatores genéticos e ambientais.Os sinais clínicos demonstrados evidenciam anormalidades envolvendo os dentes, gengivas e fissuras labiopalatais, bem como o surgimento de enfermidades secundárias, como a pneumonia, por conta da comunicação oro nasal que ocorre no caso da fenda média do palato duro. A fenda palatina é diagnosticada facilmente logo após o nascimento dos animais por conta de suas evidências clínicas, todavia faz-se necessário um exame minucioso do pacientepara detectar anomalias associadas que poderão estar presentes. $\mathrm{O}$ animal em estudo apresentou fenda palatina completa e teve uma sobrevida de três dias, a fenda deste animal possuía um comprimento total de $38,43 \mathrm{~mm}$ de extensão com o osso incisivo não fendido e ausência de fenda labial.
\end{abstract}

Palavras-chave:Fenda palatina, cavidade oral, palato duro

\section{Palatoschisis in neonatedogs Fila Brasileiro}

\begin{abstract}
The present report aims to describe the change of congenital cleft palate in a puppy of Brazilian race row with three days of life. Congenital cleft is a rare congenital anomaly in domestic animals, characterized by a defect of fusion of either primary, secondary palate or both. The pathogenesis of this disorder is still not well elucidated, however, have become aware of the many possible causes, these related to genetic and environmental factors. The demonstrated clinical signs show abnormalities involving the teeth, gums and oral clefts, as well as the emergence of secondary illnesses such as pneumonia, because of the oronasal communication that occurs as the middle of the hard palate cleft. A cleft palate is easily diagnosed shortly after birth the animals because of their clinical evidence, however, it is necessary a thorough examination of the patient to detect associated anomalies that may be present. The animal study showed complete cleft palate and had a life span of three days the slit of this animal had a total length of 38.43 $\mathrm{mm}$ in length with no split incisive bone and absence of cleft lip.
\end{abstract}

Keywords: Cleft palate, oral cavity, hard palate

\section{Introdução}

Fenda palatina, ou palatosquise, ou ainda denominada fenda secundária, é resultado da não fusão ou da fusão incompleta dos palatos duro e mole, formando uma fissura longitudinal da linha mediana no osso e na mucosa palatal, que possibilita uma comunicação oronasal (Santos \& Shimizu, 2010). Para Hoskins et al. (1997), Ribeiro \& Moreira (2005) a fenda palatina secundária é uma anomalia congênita que pode apresentar-se de modo isolada, como também de forma associada a outros defeitos congênitos orofaciais como o lábio leporino, também conhecido como anomalia do palato primário. Como já citados, os defeitos dos palatos podem acometer o palato primário, secundário ou ambos, sendo que a intensidade e a significância dos sinais clínicos irão variar conforme a severidade e a localização dos defeitos. No 
entanto, os defeitos do palato que resultam em comunicação oronasal raramente cicatrizam espontaneamente, sendo necessário o reparo cirúrgico. Ainda assim, o reparo cirúrgico pode não garantir um bom resultado na cicatrização, devido às condições naturais das cavidades orais e nasais, como presença de saliva, restos alimentares, bactérias e até mesmo a própria movimentação da língua (Dutra, 2008).

Vários são os sinais clínicos associados à fenda palatina, e dependem do grau de intensidade do defeito, podendo surgir infecções pulmonares em filhotes por aspiração de alimentos, tosse, esforços para vomitar e espirros porconta do refluxo nasal durante alimentação (Hoskins et al., 1997).

O diagnóstico em pequenos animais é realizado logo ao nascimento, sendo possível em um simples exame clínico, o que não torna necessária a realização de exames complementares como exames radiográficos, por exemplo, (San Román, 1999),. Entretanto, como pode haver coexistência com outras anormalidades congênitas, faz-se necessário um exame clínico mais detalhado (Ribeiro \& Moreira, 2005).

Hette \& Rahal (2004) descrevem a anatomia do palato em palato primário e secundário sendo o palato primário formado por lábios, crista alveolar incisiva e pré-maxila cranial ao forame incisivo e o palato secundário formado pelos ossos incisivo, maxilar e palatino. $\mathrm{O}$ autor ainda os classifica como primário e secundário, e anatomicamente como palato mole e palato duro. A localização do palato mole é caudal ao palato duro e é uma continuação do palato secundário, este é formado por tecido mole(musculatura revestida por mucosa), e quando relaxado, faz contato com a face oral da epiglote.A função dos palatos é separar fisicamente a cavidade nasal e a nasofaringe da cavidade oral e orofaringe.

Hette \& Rahal (2004) descrevem topografia das artérias palatinas maiores com origem no forame palatino maior, medial ao quarto prémolar superior e constitui o principal suprimento para o palato duro, vascularizando a mucosa oral, o periósteo e os processos alveolares. A artéria principal corre rostralmente, equidistante entre a borda lingual dos dentes e a linha média palatal, para se anastomosar com a artéria palatina maior do lado contralateral, caudalmente aos incisivos.
As artérias palatinas menores entram no palato, próximas ao último pré-molar, caudal e ligeiramente lateral ao forame palatino maior, vascularizam palato mole e porção caudal do palato duro, sendo também ramos da artéria maxilar (Hette \& Rahal, 2004). Correm caudomedialmente e se ramificam no palato duro caudal e no palato mole. O palato mole também é suprido por ramos da artéria faríngea ascendente.

As anomalias da cavidade oral e seus anexos que os cachorros apresentam ao nascer mais comumente são o lábio leporino e a fenda palatina. Ambos estão em íntima relação já que os dois provêm da mesma origem embrionário, denominando-se atualmente fenda palatina primária ou lábio leporino e fenda palatina secundário à ausência da fusão do palato duro, palato mole ou ambas as estruturas juntas (García-Arnas et al., 1991). Também é frequente que se apresentem na forma de palato fedido primário e secundário (Dutra, 2008).

Em animais o lábio leporino apresenta-se em baixa frequência e sem causar transtornos funcionais de importância, ao passo que em humanos esse defeito possui relevância estética, emocional e alimentar (Ribeiro \& Moreira, 2005, Peterson \& Kutzler, 2011). Para Fossum (2002), essa anomalia em humanos demonstra-se bastante frequente, pois de cada 650 nascidos, um apresenta-se com esse defeito, considerando a malformação crânio facial mais comum.

Os defeitos dos palatos podem acometer o palato primário, secundário ou ambos, sendo que a intensidade e a significância dos sinais clínicos irão variar conforme a severidade e a localização dos defeitos. No entanto, os defeitos do palato que resultam em comunicação oronasal raramente cicatrizam espontaneamente, sendo necessário o reparo cirúrgico. Ainda assim, o reparo cirúrgico pode não garantir um bom resultado na cicatrização, devido às condições naturais das cavidades orais e nasais, como presença de saliva, restos alimentares, bactérias e até mesmo a própria movimentação da língua (Dutra, 2008).

Diversos autores consideram incomum em pequenos animais a existência do lábio leporino, todavia tem maior frequência em raças puras do que em raças mestiças (Wiggs \& Lobprise, 1997, Hoskins et al., 1997, Dutra, 2008). Santos \& Shimizu (2010) corroboram com essa idéia e afirma que se trata de uma malformação congênita de ocorrência de baixa frequência em 
animais domésticos. Cães de raças braquicefálicas (Bulldog Francês, Pug, Boston Terrier, Pequinês, Boxer, Bulldog, ShihTzu) possuem maiores chances de nascerem com essa anomalia.

Os defeitos primários e secundários podem acometer o mesmo individuo, contudo, sabe-se que tais defeitos possuem períodos e processos de desenvolvimento embrionários diferentes e etiologia distinta (Peterson \& Kutzler, 2011).

$\mathrm{O}$ esqueleto da face bem como o tecido conectivo é formado com a migração das células da crista neural. Quando há falhas na fusão do processo frontonasal com maxilar que ocorre entre a quarta e oitava semana de desenvolvimento embrionário pode incidir acometimento do lábio fendido (Ribeiro \& Moreira, 2005).

Segundo Ribeiro \& Moreira (2005), (Dutra, 2008), ainda não é possível estabelecer um fator causal que determine o surgimento de defeitos faciais como o lábio leporino, por não haver real conhecimento etiológico e contraposição em relação ao assunto. Contudo, existem vertentes que demonstram que sua origem esteja ligada a fatores genéticos e ambientais, como deficiência de nutrientes (vitaminas B2, B6 e ácido fólico), excesso de vitamina A e D, traumatismos intrauterinos, idade avançada dos pais, consanguinidade, exposição à radiação ionizante, uso de medicamentos teratogênicos como corticoides, antifúngicos, antibióticos, antiinflamatórios e anticonvulsivantes, contato com agentes químicos (principalmente resíduos de solventes, pesticidas, herbicidas e metais) ou plantas tóxicas, fatores hormonais (esteroides), infecções, estresse, hipertermia e toxoplasmose.

Leite et al. (2005)não somente corroboram que o uso de substâncias medicamentosas parece ter grande importância para aparecimento de anomalias congênitas orofaciais, como também indicam que grupos de antibióticos, citando exemplos como as penicilinas, ampicilinas e tetraciclinas; antifúngicos, principalmente nistatina; anti-inflamatórios como diclofenaco sódico e potássico; e diferentes drogas bronco dilatadoras como fenotenol e salbutamol, com potencial ação teratogênica, pois agem no mecanismo de ação que intervém no desenvolvimento embrionário, resultando na falha parcial de fusão dos processos nasais médios, e outras anormalidades congênitas.
A persistente exposição e/ou a combinação desses fatores podem provocar modificações celulares irreversíveis alterando a formação das cristas neurais, por consequência concorrendo para mudanças no mesênquima facial, que incidirá no nascimento de indivíduos portadores de defeitos palatinos primários e/ou secundários (Ribeiro \& Moreira, 2005). Essas alterações palato faciais constituem uma condição que envolve diversos fatores, dentre os quais se destacamas suscetibilidades genéticas influenciada constantemente por fatores ambientais presentes ao longo do desenvolvimento embrionário, que interagem entre si tornando-se difícil a determinação exata do papel de um (Wiggs \& Lobprise, 1997, Dutra, 2008).

Santos \& Shimizu (2010) são vários os fatores envolvidos na patogênese das fendas palatais, sendo determinante os fatores hereditários, deficiência nutricional materna, ingestão de plantas tóxicas teratogênicas, agentes químicos e medicamentos durante a gestação. Fatores mecânicos durante o desenvolvimento embrionário condicionam o surgimento da fenda palatina adquirida. De acordo comHarvey (1987), traumas como choque elétrico, lacerações por mordeduras caninas, terapia cirúrgica e por radiação, arma de fogo e infecções crônicas graves fazem parte das principais etiologias relacionadas às fendas palatinas.

Estudos sobre artrogripose em bovinos e ovinos realizados no Brasil revelam ausência de um agente etiológico específico, sinalizando associação com fatores genéticos, sobretudo com genes autossômicos recessivos(Borges et al., 1997).

Porém, Steffens et al. (2010) relatam que algumas malformações congênitas, como as fendas orofaciais, têm relação direta com uso de medicamentos em período gestacional precoce. Ainda assim sua etiologia não se encontra totalmente esclarecida. Dentre os fatores ambientais, os medicamentos parecem ter forte influência no mecanismo que interfere no desenvolvimento embrionário, culminando na falha parcial de fusão dos processos nasais médios, decisivo para instalação da fissura palatina, bem como no aparecimento de outras anomalias de desenvolvimento.

Hette \& Rahal (2004) também descrevem que entre as possíveis causas envolvidas na patogênese das fissuras palatais congênitas em 
cães estão os fatores hereditários, as deficiências nutricionais, excesso de vitamina A e D, ingestão de medicamentos teratogênicos, terapias com corticóides, agentes químicos ou plantas tóxicas teratogênicas, interferência mecânica com o embrião, fatores hormonais, estresse emocional e o Toxoplasma gondii. Nesse caso, como há possibilidade de envolvimento hereditário, é desaconselhada à reprodução dos animais portadores dessas malformações.

Este pensamento, corrobora com o de (GarcíaArnas et al., 1991) que também menciona em seu trabalho que estes defeitos têm principalmente uma base hereditária, mas não se conhece ainda seu mecanismo etiopatológico. Sabe-se que existem numerosos fatores ambientais que podem influenciar e que os mecanismos também variam segundo a raça.

Segundo Contesini et al. (2004), as neoplasias estão entre as principais causas de lesão óssea palatina. Referem que o mastocitoma, a epúlidefibromatosa, o fibrossarcoma, o fibromeloblastoma e o osteossarcoma são as neoplasias mais frequentes. $\mathrm{O}$ tumor venéreo transmissível (T.V.T.) foi descrito por Papazoglou et al. (2001) como sendo causa de fenda palatina em dois dos seis cães presentes no seu estudo. O T.V.T. é um tumor de células redondas, cuja disseminação ocorre principalmente por via venérea ou por transplante direto de células neoplásicas (Santos et al., 2008). Apesar da sua localização mais frequente ser o aparelho genital externo de machos e fêmeas, pode apresentar outras localizações, como o tecido subcutâneo (Santos \& Shimizu, 2010) ou a cavidade nasal ou oral, justificado pelo comportamento social de lamber ou farejar a genitália externa de outros animais (Papazoglou et al., 2001, Dabus et al., 2008).

As fendas podem ocorrer no palato primário, que resulta da falha da fusão dos lábios (queilosquise), do osso incisivo (alveolosquise) ou em ambos (queiloalveolosquise), mas pode ocorrer também no palato secundário, falha de fusão do palato duro (uranosquise) e/ou no palato mole (estafilosquise) (Bojrab, 2005, Slatter, 1998, Harvey \& Emily, 1993). A afecção é herdada como traços dominantes irregulares, ou como traços recessivos (Slatter, 1998). Embora possam ocorrer defeitos primários e secundários num mesmo indivíduo, os palatos primário e secundário desenvolvem-se em períodos diferentes e independentes entre si, tratando-se de anomalias distintas (Hette \& Rahal, 2004).

Em animais o lábio leporino apresenta-se em baixa frequência e sem causar transtornos funcionais de importância, ao passo que em humanos esse defeito possui relevância estética, emocional e alimentar (Ribeiro \& Moreira, 2005, Peterson \& Kutzler, 2011). A palatosquise ou fissura na linha média do palato duro tem comunicação direta com a cavidade nasal. Por conta disso, é observada presença de células de resposta inflamatória no local. Os lóbulos pulmonares podem apresentar-se consolidados devido à presença de substâncias que fazem falsa via (Pavarini et al., 2008).

Neves et al. (2008) destacam que em humanos há além do comprometimento estético, dificuldades de fonação, distúrbios alimentares e problemas psicológicos. Da mesma forma que em humanos, os animais podem desenvolver problemas de dentição relacionados à erupção, desenvolvimento, número e tamanho, tanto na dentição decídua como na permanente.

$\mathrm{Na}$ cavidade abdominal há inexistência de conteúdo estomacal, presença de gases nas alças intestinais e não expulsão do mecônio em neonatos, estando este presente na ampola retal. $\mathrm{O}$ fato de não haver presença de alimento no estômago é explicado por conta do animal não conseguir mamar, isto é possível por não existir pressão negativa na boca suficientemente capaz para haver sucção, o que rapidamente deixa o animal em um quadro de caquexia, possibilitando a morte do mesmo por inanição por não haver reservas nutricionais para sua manutenção (Hette \& Rahal, 2004). A fissura palatina por possibilitar a comunicação oronasal favorece a aspiração de alimentos juntamente com microrganismos presentes na cavidade oral para os pulmões, resultando no desenvolvimento de pneumonia, também considerada uma importante causa de morte. A caquexia pode aparecer em decorrência de todo o processo de inanição, possibilitando a supressão imunológica, reduzindo as respostas imunocelulares e humoral, predispondo também à pneumonia, o que poderá levar facilmente o animal a um quadro de insuficiência respiratória (Santos \& Shimizu, 2010).

Para Nelson \& Slatter (1998), o diagnóstico da afecção é realizado durante o exame físico observando diretamente a cavidade oral do paciente. Mediante o exame clínico da cavidade 
oral é possível a fácil visualização e deteç̧ão do problema. Com um exame radiográfico visualizase a separação completa dos ossos palatinos. Fendas palatinas primárias ficam evidentes por ocasião do nascimento do animal, como fissura anormal no lábio superior. Estes animais devem ser examinados em busca de fendas coexistentes do palato secundário (palatos duro e mole) (Slatter, 1998, Fossum, 2002, Hette \& Rahal, 2004). Fendas do palato secundário frequentemente passam despercebidas até o aparecimento dos sintomas, como crescimento deficiente; drenagem de leite pelas narinas durante ou depois de mamar; tosse, engasgamentos, espirros durante a ingestão dos alimentos e infecção do trato respiratório (rinite, laringotraqueíte e pneumonia por aspiração) (Hoskins, 1997, Slatter, 1998, Fossum, 2002, Hette\& Rahal, 2004).

Por ocasião da fácil visualização da fissura labial que tanto pode acometer o lábio superior (mais comum) ou lábio inferior, a fenda do tipo primário (lábio leporino) é facilmente diagnosticada. A palatosquise ou fenda do tipo secundário, diversas vezes pode não obter um diagnóstico precoce, sendo percebida somente por ocasião do aparecimento de sinais clínicos relacionados com a afecção, como engasgamentos, tosse, espirros, refluxo de leite pelas narinas no momento da alimentação, bem como o surgimento de infecções das vias respiratórias (Nelson, 1998).

Segundo Dutra (2008), o diagnóstico diferencial deve levar em conta as fendas adquiridas ou traumáticas, rinite e corpo estranho nasal, entre outros.

O tratamento adotado para esta afecção é a correção cirúrgica, que imprescindivelmente deve ser levada em consideração as condições em que se encontra o paciente. Para que ocorra uma resposta satisfatória do tratamento cirúrgico, de medidas terapêuticas e estabelecimento de um aporte nutricional favoráveis, sente-se a necessidade de que ocorra uma identificação precoce dessas lesões. Existem diversas técnicas para correção desses defeitos palatofaciais. Entretanto, para o sucesso da correção desses defeitos, é aconselhável que o animal acometido deve ter idade e estado nutricional adequados para suportar protocolos anestésicos. Tendo isso em vista, é relevante que em fases iniciais da vida do animal sua alimentação seja procedida via sonda oroesofágica (Hette \& Rahal, 2004).
Segundo Bojrab (1990) a indicação do procedimento cirúrgico ocorre quando a abertura oronasal não permite seu fechamento adequadamente, e sua extensão é suficientemente o bastante para permitir a passagem de alimentos e fluídos para a cavidade nasal. Para Dutra (2008), a correção da fenda palatina através do procedimento cirúrgico em cães é indicada somente quando o animal completar sete ou oito semanas de vida, o que proporciona ao cirurgião um maior campo cirúrgico e tecidos menos friáveis.

Os tecidos devem ser suavemente manipulados para manter o suprimento sanguíneo preservado e evitar esmagamento ou ressecamento dos tecidos moles, que se danificam facilmente. Deve-se evitar o uso de eletrocoagulação para hemostasia, pois este instrumento pode causar necrose, predispondo à deiscência. O muco periósteo do palato duro é suprido principalmente pelo par de artérias palatinas principais, então sempre que for necessária a utilização de um "flap" de muco periósteo deverá ser incorporado neste uma artéria palatina principal intacta (Bojrab, 1996).

As áreas de onde os "flaps" e incisões relaxantes são retiradas devem ser deixados que cicatrizem por segunda intenção $o$ que geralmente ocorre dentro de três semanas (Fossum, 2002).

A reparação do defeito palatino é feita com a própria mucosa palatina, também chamada no caso do palato duro de uranorrafia e no palato mole de estafilorrafia (Gioso, 2003).

Alguns princípios devem ser seguidos na correção dos defeitos como: não haver tensão na linha de sutura, suturar superfícies cruentas entre si, evitar incisar as artérias palatinas maiores e menores (Gioso, 2003, Harvey \& Emily, 1993)

Em animais a queiloplastia (correção cirúrgica da fissura labial) é de caráter sumariamente estético, por não haver interferência funcional (Gioso, 2003).

De acordo com Ribeiro \& Moreira (2005), animal acometido, somente deve passar por procedimento cirúrgico, quando este obtiver condições adequadas para serem submetidos à anestesia geral. No procedimento de queilopastia é criado um retalho de mucosa a fim de obter tecido suficiente para a reconstrução labial e divisão definitiva da cavidade oral e nasal. $\mathrm{O}$ retalho da mucosa nasal deve ser sutura em 
padrão z modificado ao retalho da mucosa oral. Com o fechamento da fissura labial há a necessidade uma distância da narina ventral à borda ventral livre do lábio.

Segundo Smith (2000) para o sucesso da cirurgia, é de suma importância a preservação da estrutura vascular dos enxertos, e obter a capacidade desses enxertos resistirem aos efeitos mecânicos naturalmente exercidos pelo próprio animal como mastigação, deglutição e outros movimentos exercidos pela a língua. $\mathrm{O}$ prognóstico é favorável nos pacientes com pequenas fendas ou nos pacientes em que são corrigidas cirurgicamente, pois se elimina o risco de aspiração de alimentos para a via respiratória (Silva \& Sousa, 2006).

Animais que não sejam tratados cirurgicamente geralmente são eutanasiados ou acabam por morrer devido a pneumonia por aspiração (Corrêa, 2008). Os animais submetidos à cirurgia alcançam altas taxas de insucessos no pós-operatório, isto é devido principalmente em decorrência da deiscência de sutura que ocorre entre o sétimo e décimo dia (Souza et at., 2007). Para Smith (2000) e Griffiths \& Sullivan (2001), o insucesso da cirurgia é devido principalmente a alta tensão exercida no local no momento da sutura, culminando no processo de deiscência.

A cronicidade do defeito também é um dos fatores preponderantes, como também o estado de saúde do animal (Souza et al., 2007) e a irritação e inflamação causadas pelo movimento da língua sobre a ferida cirúrgica, como também o fato de pacientes terem acesso ao exterior de suas casas e a alimentos grosseiros e rígidos em maioria dos casos também contribui para a recidiva da fenda palatina no pós-operatório (Leeet al., 2006).

Ainda assim, as complicações da cirurgia são minimizadas se forem considerados princípios básicos da cirurgia do palato, como refere Sivacolundhu (2007). Estes incluem: realizar enxertos de dimensões um pouco maiores que o defeito, manter a vascularização do enxerto, reavivar os bordos do tecido a suturar, evitar colocar as suturas sobre o defeito e manipular o tecido delicadamente (Silva \& Sousa, 2006).

\section{Material e Métodos}

Foram dissecados dois filhotes machos com três dias de nascimento da raça Fila Brasileiros (com Registro genealógico) de uma ninhada de dez filhotes, estes tiveram óbito natural por esmagamento, sendo que somente um apresentava fenda palatina congênita, o outro filhote foi utilizado apenas para estudo comparativo. Para a realização deste, também contamos com uma anamnese detalhada por parte do criador, incluindo o histórico reprodutivo da genitora.

Para a dissecação utilizou-se uma lupa circular de luz fria, bisturi número 4 com lâmina 24, pinças anatômicas e costótomo. Com auxílio das pinças e do bisturi fez-se uma secção horizontal em sentido crânio-caudal partindo da comissura labial direita, incisando apele, o músculobucinador e o músculo masseter. No mesmo sentido seccionou-se o ramo da mandíbula horizontalmente utilizando um costótomo. Em seguida este mesmo procedimento foi realizado no antímero esquerdo partindo da comissura labial esquerda. Continuou-se a dissecação incisando pele e o músculo masseter, o músculo genioióideo e o músculo miloióideo, em um corte transversal entre as secções dos ramos mandibulares.

Posteriormente foi incisada a base da língua e retirado todo osso mandibular para total exposição da região palatina. Avaliaram-se as estruturas da cavidade oral, direcionando-se para a região anatômica de interesse, o palato. No filhote acometido foi mensurada a fissura palatal em sentido longitudinal com auxílio de um paquímetro digital. Utilizando o mesmo instrumento, realizou-se também a mensuração da extensão do palato íntegro.Também realizou o registro fotográfico digital com auxílio de uma máquinafotográfica digital de 12 megapixels e flash automático, sustentada em tripé.

\section{Resultados e Discussão}

A fenda palatina encontrada no filhote em estudo apresentava-se por toda a extensão longitudinal do palato, medindo $38,43 \mathrm{~mm}$, sendo, classificada como fenda palatina do tipo secundário ou fenda palatina completa, ocasionada pela ausência da fusão do palato duro, palato mole ou ambas as estruturas juntas, conforme preconizado por (García-Arnas et al., 1991). O palato íntegro do segundo filhote mediu 40,34mm, confirmando assim a normalidade na formação dos demais ossos cranianos (Figura 1A e B).

Ribeiro \& Moreira (2005) e Dutra (2008), citam em seus trabalhos que ainda não é possível determinar um fator causal. Todavia, estabelecem 
alguns fatores envolvidos como a genética e a consanguinidade, fator este presente no filhote estudado, pois seus genitores eram filhos do mesmo pai, o que possivelmente tenha contribuído para a existência desta afecção, contudo o criador informou que se tratava da segunda ninhada sendo que a primeira foi de cinco filhotes sem nenhum tipo de alteração e a segunda ninhada possuía 10 filhotes e apenas um apresentou esta anomalia.

Hette \& Rahal (2004) descrevem a ausência de conteúdo estomacal que é explicada por conta do animal não conseguir mamar, isto é possível por não existir pressão negativa na cavidade oral propriamente dita, que seja capaz de culminar em sucção, o que rapidamente deixa o animal em um quadro de caquexia, possibilitando a morte do mesmo por inanição, com base na anamnese o proprietário relatou que o desenvolvimento deste filhote estava comprometido e que iniciou a alimentação artificial com sucedâneo de leite, administrado pela cavidade oral com auxilia de uma seringa, porém não observou a melhora do filhote. Corrêa (2008) afirma que animais não tratados cirurgicamente geralmente são eutanasiados ou acabam por morrer devido a pneumonia por aspiração, diferindo do relato do proprietário que afirma ter encontrado o animal morto sob a mãe junto com o outro exemplar, sugerindo óbito por esmagamento, porém não podemos descarta a morte por inanição com esmagamento posterior, uma vez que o proprietário não observava a ninhada em período integral.
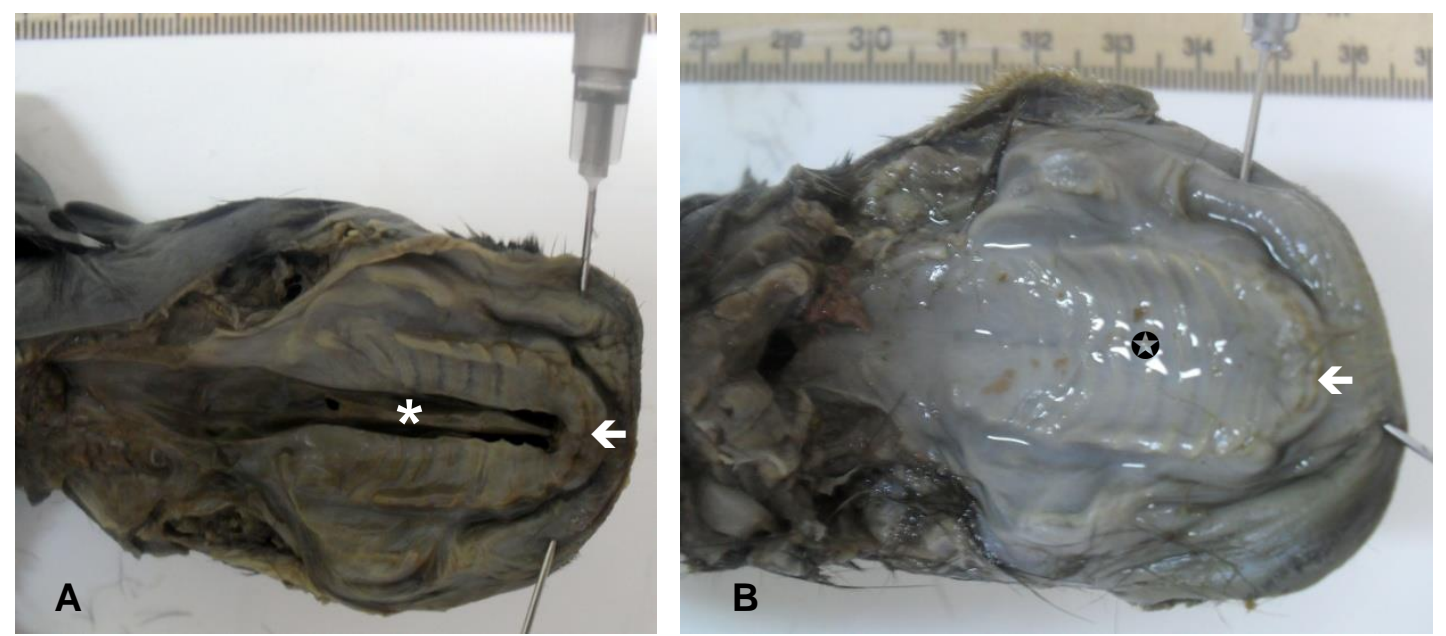

Figura 1. Em A observa-se a fenda palatina secundária (*) comunicando a cavidade oral propriamente dita com a cavidade nasal, e a região incisava íntegra (૯), $\mathbf{B}$ demonstra uma região palatina normal

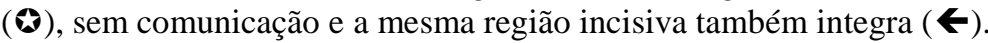

\section{Conclusão}

Alterações morfológicas em animais domésticos ainda não possuem uma descrição detalhada de sua origem, estudos descritivos não são capazes de elucidar sua origem, porém podem contribuir para um melhor entendimento de suas consequências no desenvolvimento pósnatal bem como gerar subsídios para o aprimoramento de técnicas cirúrgicas para propiciar uma maior sobrevida a animais acometidos por estas teratologias.

\section{Referências bibliográficas}

Bojrab, M. J. 2005. Técnicas atuais em cirurgia de pequenos animais. Editora Roca,São Paulo.
Borges, A. S., Mendes, L. C. N., Vasconcelos, R., Alves, A. L. G. \& Rodrigues, C. A. 1997. Espinha bifida e mielodisplasia em ruminantes: Relato de caso. Arquivo Brasileiro de Medicina Veterinária $e$ Zootecnia, 49, 685-692.

Contesini, E. A., Pippi, N. L., de Castro Beck, C. A., Brun, M. V., Costa Leme, M., Raffi, M. B., Godoy, C. L. B., Bonfada, A. T., Gomes, K. \& Trindade, A. B. 2004. Cartilagem homóloga conservada em glicerina para restauração de fenda de palato duro experimental em cães. Revista da FZVA, 11.

Corrêa, A. C. 2008. Técnica de retalhos sobrepostos em fenda palatina secundária em cão - Relato de caso. Associação Nacional de 
Clínicos Veterinários de Pequenos Animais de São Paulo e Universidade Anhembi-Morumbi.

Dabus, D. M. M., Tentrin, T. C., Bocardo, M., Lima, G. S., Lot, R. F. E., Barian, M. H. \& Rocha, N. S. 2008. Estudo epidemiológico do tumor venéreo trnsmissível baseado nos padrões plasmocitóide e linfocitóide em cães atendidos no hospoital veterinário da faculdade de medicina veterinária e zootecnia de Garça. Revista Científica Eletretrônica de Medicina Veterinária, 6, 1-7.

Dutra, A. T. 2008. Defeitos palatinos congênitos. Medicina Veterinária. Universidade Castelo Branco.

Fossum, T. W. Cirurgia de Pequenos Animais. 1. ed. São Paulo: Mosby, p.1606, 2002.

García-Arnas, F., Llorens, M., San Román Ascaso, F., Prandi Chevalier, D. \& Peña Giménez, M. 1991. Palatosquisis en la especie canina. Clínica veterinaria de pequeños animales.

Gioso, M. A. 2003. Odontologia para o clínico de pequenos animais, 5a edn. Leditora,São Paulo.

Harvey, C. E. 1987. Palate defects in dogs and cats. Point Veterinaire, 9, 404-418.

Harvey, C. E. \& Emily, P. 1993. Small animal dentistry,St. Louis: Mosby.

Hette, K. \& Rahal, S. C. Defeitos congênitos do palato em cães: revisão da literatura e relato de três casos. Clínica Veterinária, São Paulo: Guará, v. 9, n. 50, p. 30-40, il., color. maio/jun. 2004.

Hoskins, J., Dimski, D. \& Hoskins, D. 1997. O sistema digestivo. Pediatria veterinária 3/4 cães e gatos do nascimento aos seis meses, 2, 120-171.

Lee J., Kim, Y., Kim, M., Lee, J., Choi, J., Yeom, D., Park, J.\& HONG, S. Application of a temporary palatal prosthesis in a puppy suffering from cleft palate. Journal of Veterinary Science, v.7, n.1, p.93-95, 2006.

Leite, I. C. G., Paumgartten, F. J. R. \& Koifman, S. 2005. Fendas orofaciais no recém-nascido e o uso de medicamentos e condições de saúde materna: estudo caso-controle na cidade do Rio de Janeiro, Brasil. Revista Brasileira de Saúde Maternal infantil, 5, 35-43.
Nelson, A. W. \& Slatter, D. 1998. Sistema respiratório superior. In: Slatter, D. (ed.) Manual de cirurgia de pequenos animais. Manole, São Paulo.

Neves, A. C. C., Monteiro, A. M. \& Ng, H. G. 2008. Prevalência das fissuras labiopalatinas na Associaçao de Fissurados Labiopalatinos de Sao José dos Campos/SP. Revista Biociências, 8, 69-74.

Papazoglou, L. G., Koutinas, A. F., Plevraki, A. G. \& Tontis, D. 2001. Primary intranasal transmissible venereal tumour in the dog: a retrospective study of six spontaneous cases. Journal of Veterinary Medicine Series A, 48, 391-400.

Pavarini, S. P., Sonne, L., Antoniassi, N. A. B., Santos, A. S., Pescador, C. A., Corbellini, L. G. \& Driemeier, D. 2008. Anomalias congênitas em fetos bovinos abortados no sul do Brasil. Pesquisa Veterinária Brasileira, 28, 149-154.

Peterson, M. E. \& Kutzler, M. 2011. Small animal pediatrics: the first 12 months of life. Elsevier Health Sciences.

Ribeiro, E. M. \& Moreira, A. S. C. G. 2005. Atualização sobre o tratamento multidisciplinar das fissuras labiais e palatinas. Revista Brasileira em Promoção da Saúde, 18, 31-40.

San Román, F. 1999. Atlas de odontologia de pequenos animais. São Paulo, 1, 217-241.

Santos, D. E., Silva, D. T., Toledo-Pinto, E. A. \& Lot, R. F. E. 2008. Tumor venéreo transmissível (TVT): Revisão de literatura. Revista Científica Eletrônica de Medicina Veterinária, 110, 1-7.

Santos, P. C. G. \& Shimizu, F. A. 2010. Aspectos anatomo histopatológico do tumor venéreo transmissível. Revista Científica Eletrônica de Medicina Veterinária, 3, 1-4.

Silva, M. R. \& Sousa, A. G. A. 2006. Redução de fenda palatina secundária em um gato. Ciência Veterinária nos Trópicos, 9, 97-101.

Sivacolundhu, R. K. 2007. Use of local and axial pattern flaps for reconstruction of the hard and soft palate. Clinical Techniques in Small Animal Practice, 22, 61-69.

Slatter, D. H. 1998. Manual de cirurgia de pequenos animais. Manole,São Paulo. 
Smith, M. M. 2000. Oronasal fistula repair. Clinical techniques in small animal practice, 15, 243-250.

Souza, H .J .M., Alffeld, V .F., Cicarella, L. C., Grilo, J. C. \& Castelan, F. G. Oclusão de fístula oronasal crónica utilizando a "U"Plastia da mucosa palatal em gato. Acta Scientiae Veterinariae, v.35, (Supl 2), p.474475, 2007.

Steffens, H., Brandão, A. A., Guariza Filho, O., Grégio, A. M. T. \& Steffens, J. P. 2010. Avaliação do potencial do cloranfenicol para induzir teratogenicamente o aparecimento de fissura palatina em ratos Wistar. Revista Sul Brasileira de Zootecnia, 7, 154-158.
Wiggs, R. B. \& Lobprise, H. B. 1997. Veterinary Dentistry: principles and practice. LippincottRaven Publishers.

Recebido em Julho 23, 2014

Aceito em Setembro 03, 2014

License information: This is an open-access article distributed under the terms of the Creative Commons Attribution License, which permits unrestricted use, distribution, and reproduction in any medium, provided the original work is properly cited. 